\title{
Dental organisations support Antibiotic Awareness Week
}

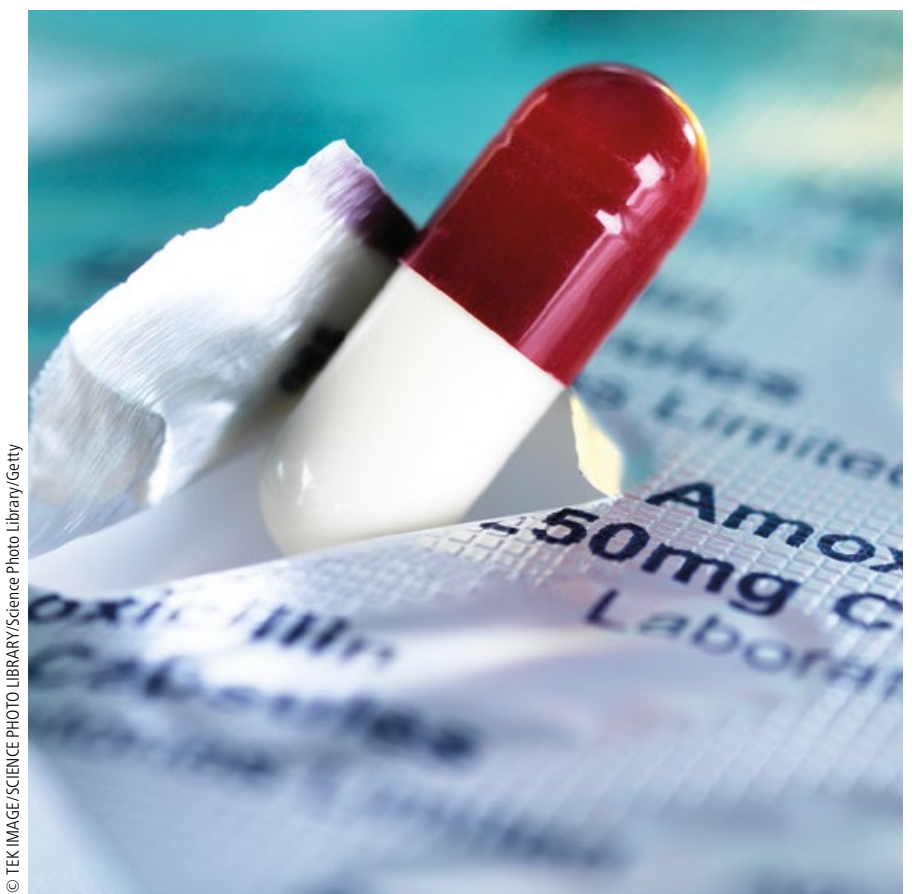

National dental organisations came together last month to support the World Health Organisation's Antibiotic Awareness Week, which ran from 18-24 November.

The Faculty of General Dental Practice UK, Association of Clinical Oral Microbiologists, College of General Dentistry, British Dental Association, British Association of Oral Surgeons, Association of Dental Hospitals and the dental sub-group of the Scottish Antimicrobial Prescribing Group are repeating the message to patients that 'antibiotics do not cure toothache', and reminding dental teams that antibiotics should only be used as an adjunct to definitive clinical management of the cause, and only where indicated.

Since the start of the COVID-19 pandemic, the dental profession has focused on preventing the transmission of SARS-CoV-2 to their team, patients and the wider population. During the first national lockdown, the provision of general dental services was severely restricted and limited to the referral of urgent and emergency cases and the provision of advice, analgesics and antimicrobials where appropriate.

As a result of these constraints, a marked increase in antibiotics prescribed in NHS general dental practice has been reported. In England the number of prescriptions was up 22\% during April to June 2020, and in Scotland there were steady month-on-month increases from April, peaking at a 50\% rise in July, when compared with the same periods in 2019.

It is estimated that dentists account for around $10 \%$ of all oral antimicrobial agents prescribed in healthcare, and a clear link has been established between the consumption of antimicrobial agents in human medicine, animal healthcare and agriculture and increasing rates of antimicrobial resistance.

While antimicrobial resistance continues to be a long-term problem, a government review reported that 700,000 people worldwide are already dying of antibiotic-resistant infections every year, and estimated that by 2050 the annual global cost could be 10 million lives and $£ 66$ trillion.

Prudent prescribing of antimicrobials can slow down the further development of antimicrobial resistance, and all healthcare prescribers play a vital role. The dental profession has shown its clear commitment in addressing antimicrobial resistance by significantly reducing the use of antibiotics in dental practice over the last decade.

Last year alone, dentists in the UK reduced their prescribing of antimicrobials by $9 \%$, and the organisations say it is vital that the profession maintains and builds on this success. They are encouraging all dentists to continue to play their part in reducing the development of antimicrobial resistance by ensuring appropriate prescribing of antibiotics when managing their patients during the COVID-19 pandemic and into the future.

Guidelines for antimicrobial prescribing in dentistry are available at https://www.fgdp.org.uk/guidance-standards/antimicrobialprescribing-gdps and https://bnf.nice.org.uk.

Posters and leaflets for patients are available at https://www. fgdp.org.uk/antimicrobial-prescribing, and a Dental Antimicrobial Stewardship Toolkit, developed by FGDP(UK), the British Dental Association and Public Health England, is accessible at https://www. gov.uk/guidance/dental-antimicrobial-stewardship-toolkit.

\section{GDC and CQC issue statement on life-support training}

The General Dental Council (GDC) and Care Quality Commission (CQC) have issued a joint statement on life-support training for dental professionals in light of the COVID-19 pandemic.

The Resuscitation Council (UK) recommends cardiopulmonary resuscitation (CPR) training for dental teams. Their document Quality Standards: Primary Dental Care (https:// bit.ly/2JrPZfR) states: 'Dental practitioners and other dental healthcare staff should update their knowledge and skills in resuscitation at least annually'.

The GDC and CQC said: 'Together, we recognise that COVID-

19 social distancing can make accessing accredited training difficult. This includes Basic Life Support (BLS) and Immediate Life Support (ILS).

'Providers and individual dental professionals should continue to make every effort to complete accredited BLS/ILS training annually. However, if this training is unavailable due to current COVID-19 restrictions, appropriate alternative provisions, in accordance with relevant government guidance, should be made, for example: e-learning, self-directed study, team-based scenario training in the clinical environment.

'Records and evidence of efforts made to access training and to update CPR knowledge and skills should be maintained.' 